\title{
Computation of the Soft Anomalous Dimension Matrix in Coordinate Space
}

\author{
Alexander Mitov, George Sterman, Ilmo Sung \\ C.N. Yang Institute for Theoretical Physics, Stony Brook University, Stony Brook, New York 11794-3840, USA
}

(Dated: November 5, 2018)

\begin{abstract}
We complete the coordinate space calculation of the three-parton correlation in the two-loop massive soft anomalous dimension matrix. The full answer agrees with the result found previously by a different approach. The coordinate space treatment of renormalized two-loop gluon exchange diagrams exhibits their color symmetries in a transparent fashion. We compare coordinate space calculations of the soft anomalous dimension matrix with massive and massless eikonal lines and examine its nonuniform limit at absolute threshold.
\end{abstract}

\section{INTRODUCTION}

As a result of recent investigations, our knowledge of the infrared (IR) singularities of massive gauge amplitudes has progressed from one [1, 2] to two loops [3 [8]. We are now in a position to predict the single IR poles of any two-loop amplitude with arbitrary numbers of partons of arbitrary masses. This helps determine the behavior of amplitudes close to kinematic thresholds, and we now have available the two-loop input necessary for threshold resummation to next-to-next-to leading logarithm for heavy quark production [7 9]. Much of the new information is encoded in the two-loop anomalous dimension matrices for coupled massive and/or massless partons, which we refer to collectively as the "massive soft anomalous dimension matrix" [3] 8$]$.

A goal of the present paper is to clarify the relationship between two calculations of a key component in the twoloop massive anomalous dimension matrix, the color antisymmetric three-parton correlation. Implicit expressions were presented for these correlations in [4], partly in terms of integrals in Euclidean space. Subsequently an elegant analytic expression was presented in Ref. [6]. In Ref. [6] calculations were carried out in momentum space, and Ref. [4] in position space. Of course, these two approaches should give equivalent results, and we show below that when all contributions are taken in account, they indeed do. Part of our motivation in presenting the details leading to this expected outcome is that an apparent discrepancy in the calculations was raised in [6]. This involves the diagrams called "double exchange" in Ref. [4] and "planar" in [6], illustrated here in Fig. 1] As we shall see, this apparent disagreement arises simply because Ref. [4] exhibited results for these diagrams before renormalization. We present the remaining analysis here not as new results, but to confirm the equivalence of the two calculations, and because results of Ref. [4] were used in deriving the expression for the total cross section given in [8]. Of particular relevance was the conclusion that the two-loop massive anomalous dimension matrix is diagonal in the $s$-channel singlet-octet basis for pair production from incoming light quarks or gluons at ninety degrees in the partonic center of mass.

We will show in Sec. II that apparent discrepancies between results presented in Refs. [4] and [6] are entirely due to two-loop diagrams with one-loop counterterms. These diagrams, which were not presented in [4], do not affect the color diagonalization of the anomalous dimension matrix at ninety degrees. This diagonalization was indeed confirmed in [6]. It is of interest to see the simplicity of the coordinate space analysis, which may have applications at higher orders 10 12].

In Sec. III, we discuss similarities and differences encountered in the coordinate space calculations when all lines are massless. Here, we present a discussion in which both one- and two-loop massless integrals are regularized dimensionally, and reproduce from a purely coordinate space analysis the absence of three-eikonal color correlations, a result found in [13], which relied on arguments in both momentum and coordinate space.

Finally, in Sec. [V] we discuss the limit of absolute threshold (pair creation at rest). Reference [6] discovered a nonuniform limit of the anomalous dimension matrix at absolute threshold, when expressed in terms of the scattering angle. Here we rederive the result found Ref. [6], using the methods introduced in Ref. [4]. We show how the singularity structure of the integrals restricts the non-uniform limit to a region of momentum space where the eikonal approximation fails. This limit of the soft anomalous dimension matrix is not directly relevant to the total pair production cross section [7, 8]. For completeness, explicit expressions for the relevant integrals are exhibited in an appendix.

\section{TWO-LOOP DOUBLE EXCHANGE DIAGRAMS}

The formalism for the computation of soft anomalous dimension matrices has been discussed extensively in Refs. [1] and elsewhere. The relevant diagrams consist of radiative corrections to incoming and outgoing partonic propagators in eikonal approximation. The resulting diagrammatic expressions are scaleless, and vanish in dimensional regularization. 


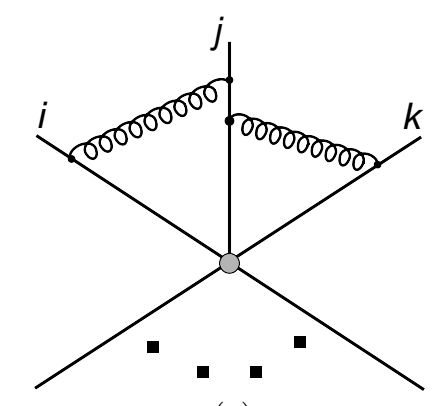

(a)

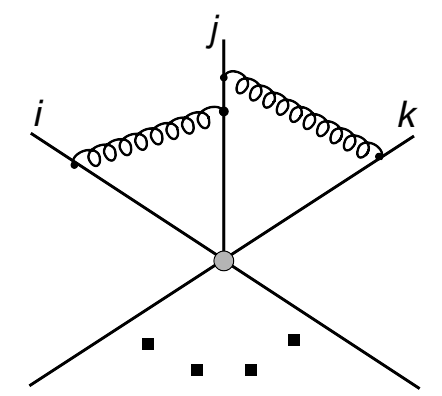

(b)

FIG. 1: Double exchange diagrams discussed in the text. The shaded circle represents an $n$-eikonal vertex.

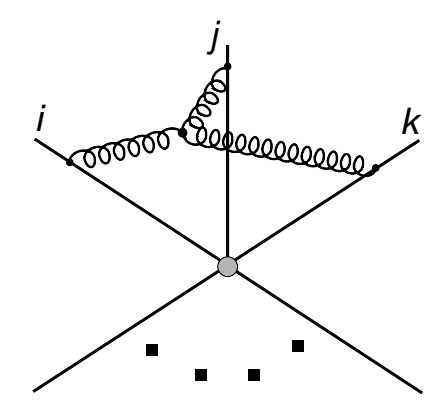

FIG. 2: Non-planar diagram for the two-loop anomalous dimension matrix.

Their ultraviolet poles, however, define counterterms and hence the anomalous dimensions we are after 1 , 13]. In this formalism, we factorize the full eikonal amplitude into a soft function, with one logarithmic singularity per loop and all nontrivial color exchange, and color-diagonal jet functions. This factorization ensures that in the limit of vanishing eikonal masses the soft function is free of collinear singularities [13, 14]. A natural generalization of this procedure to eikonals of arbitrary mass is the scheme employed in Ref. [8], in which the jet functions are defined as the square roots of the low-mass limits of the corresponding form factors [15, 16]. ${ }^{1}$

The most challenging two-loop contribution to the massive anomalous dimension matrix is the set of non-planar diagrams with a three-gluon vertex connecting three eikonals, of the type shown in Fig. 2. Although these diagrams are rather complex, they have no UV-divergent subdiagrams. It was shown in Ref. [13] by a momentum-space change of variables, that the ultraviolet poles of these diagrams vanish when all three of the interacting eikonals are massless. This result was rederived in Ref. [4] in coordinate space and extended to diagrams with two massless interacting eikonals, while a manifestly nonvanishing integral representation was given for the UV pole when all three interacting eikonals are massive. An analytic expression for the "non-planar" diagrams like Fig. 2 with massive external partons was derived in Ref. [6]. Surprisingly, it turned out to be very simple (see appendix). In numerical tests, it agrees with the integral representation given in Ref. [4].

In this section, we revisit the double-exchange diagrams of Fig. 11in coordinate space, including their renormalization. As explained in Ref. [4] and confirmed below, the contributions from the two-loop double exchange diagrams are fully symmetric in color after combining pairs like those shown in Fig. 1 They contain, however, subdiagrams with UV poles, and thus require one-loop counterterms. These counterterms were not considered explicitly in Ref. [4]. In Fig. 3 we show the renormalization of the double exchange diagrams of Fig. 1, with divergent loops replaced by counterterms. In Fig. 3 a , for example, the counterterm depends on the pair of momenta $p_{k}, p_{j}$ and the remaining one-loop diagram on $p_{i}$ and $p_{j}$.

To determine the counterterms in our coordinate space formalism, and to establish notation, we review the oneloop case, illustrated in Fig. 4. The counterterms, in turn, are found from the poles of one-loop diagrams. Following the notation of Ref. [4] (see also Appendix B of Ref. [13] for the same calculation in slightly different notation), we

${ }^{1}$ We note that Ref. [6] analyzes the massive amplitude directly, without subtractions, but direct comparison can still be made, because by construction subtractions do not affect color exchange. 


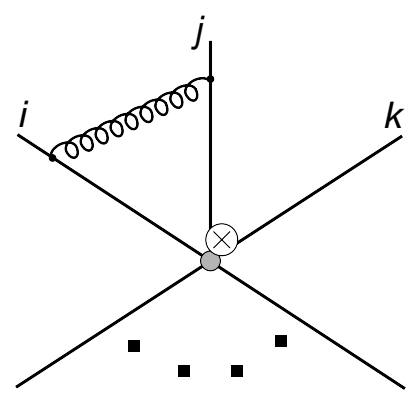

(a)

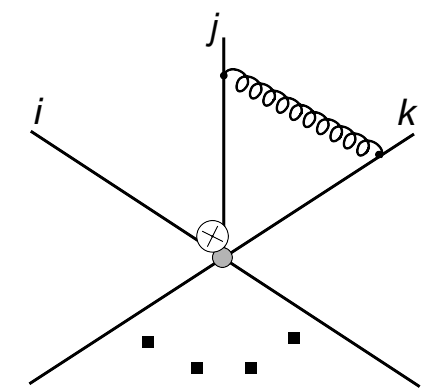

(b)

FIG. 3: Order $g^{4}$ diagrams containing one-loop UV counterterm.

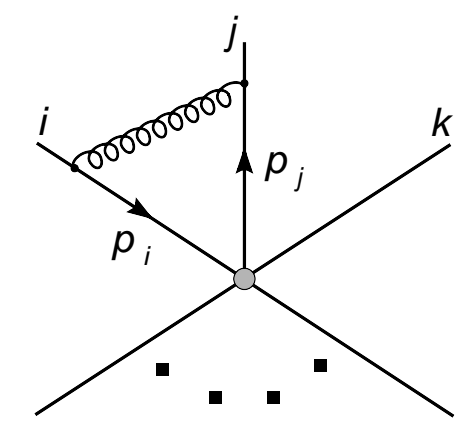

FIG. 4: A gluon exchanged between an incoming quark $i$ and an outgoing quark $j$ that contributes to Eq. (2).

introduce the scalar propagator in coordinate space,

$$
\begin{aligned}
\Delta(x-y) & \equiv i \int d^{4-2 \varepsilon} k e^{-i k \cdot(x-y)} \frac{1}{k^{2}+i \epsilon} \\
& =-\frac{\Gamma(1-\varepsilon)}{4 \pi^{2-\varepsilon}} \frac{1}{\left((x-y)^{2}-i \epsilon\right)^{1-\varepsilon}} \\
& \equiv-c(\varepsilon) \frac{1}{4 \pi^{2}} \frac{1}{\left((x-y)^{2}-i \epsilon\right)^{1-\varepsilon}},
\end{aligned}
$$

where the third relation defines the constant $c(\varepsilon)=1+\mathcal{O}(\varepsilon)$.

The coefficient of $\alpha_{s} / \pi$ in the one-loop correction corresponding to Fig. 4 is a matrix in color space, ${ }^{2}$

$$
\begin{aligned}
\mathcal{M}^{(1)}\left(p_{i}, p_{j}, \varepsilon\right) & \left.=c(\varepsilon)\left(\mathbf{T}_{i} \cdot \mathbf{T}_{j}\right) \int_{0}^{\infty} d \lambda_{j} \int_{0}^{\infty} d \lambda_{i} \frac{p_{i} \cdot p_{j}}{\left[\left(\lambda_{j} p_{j}-\lambda_{i} p_{i}\right)^{2}-i \epsilon\right]}\right]\left.^{1-\varepsilon}\right|_{U V} \\
& =\left.c(\varepsilon)\left(\mathbf{T}_{i} \cdot \mathbf{T}_{j}\right)\left(p_{i} \cdot p_{j}\right) \int_{0}^{\infty} \frac{d \lambda_{j}}{\lambda_{j}^{1-2 \varepsilon}} \int_{0}^{\infty} \frac{d \sigma}{\left[\left(p_{j}-\sigma p_{i}\right)^{2}-i \epsilon\right]}\right|_{U V} \\
& =c(\varepsilon) m_{j}^{2 \varepsilon}\left(\mathbf{T}_{i} \cdot \mathbf{T}_{j}\right) \frac{1}{2 \varepsilon} I^{(-1)}\left(p_{i}, p_{j}\right) .
\end{aligned}
$$

The color factors associated with the gluon exchange are represented in the basis-independent notation introduced in Ref. [17] and generally employed in analyses of soft anomalous dimensions. The final relation in Eq. (22) shows the contribution of the UV pole to the one-loop anomalous dimension matrix in terms of the function

$$
\begin{aligned}
I\left(p_{i}, p_{j}, \varepsilon\right) & \equiv\left(p_{i} \cdot p_{j}\right) m_{j}^{-2 \varepsilon} \int_{0}^{\infty} d \sigma \frac{1}{\left(m_{j}^{2}-2 \sigma p_{i} \cdot p_{j}+\sigma^{2} m_{i}^{2}-i \epsilon\right)^{1-\varepsilon}} \\
& =I^{(-1)}\left(p_{i}, p_{j}\right)+\varepsilon I^{(0)}\left(p_{i}, p_{j}\right)+\mathcal{O}\left(\varepsilon^{2}\right),
\end{aligned}
$$

\footnotetext{
2 This factor is referred to as the "velocity factor" in Ref. [13]. Because all partonic lines are eikonal, we need not distinguish between velocities and momenta for the external lines.
} 
with $m_{i, j}^{2}=p_{i, j}^{2} \neq 0$. The massless limit is explored in Sec. III. For the purposes of this discussion, we may take $-p_{j} \cdot p_{i}>0$, corresponding to one line incoming and the other outgoing. In this case, $I\left(p_{i}, p_{j}, \varepsilon\right)$ is real. The explicit results for the functions $I^{(-1,0)}\left(p_{i}, p_{j}\right)$ for both signs of the invariant are given in the appendix.

It is important to note that the prefactor in Eq. (2) is arbitrary at $\mathcal{O}(\varepsilon)$, because it reflects the choice we make to define the integral over $\lambda_{j}$, a variable with dimensions of length squared. In effect, our choice for these functions constitutes a minimal subtraction scheme. In particular, the factor $m_{j}^{2 \varepsilon}$ in (2) and following expressions is kept as a bookkeeping device only, because it matches the definition of $I\left(p_{i}, p_{j}, \varepsilon\right)$. For the purposes of calculating anomalous dimensions it is only necessary that the prefactors for all such integrals be defined in the same way. Then, as we will see below, overall factors like $c(\varepsilon)$ and $m_{j}^{2 \varepsilon}$ will not affect our results for the dimensionless pole terms of $\mathcal{M}^{(1)}$.

The one-loop soft anomalous dimension matrix is defined only up to color-diagonal contributions associated with its eikonal lines, which absorb collinear logarithms and are factorized from the soft function. In any such factorization scheme, the one-loop anomalous dimension matrix is found from the residues of the poles $1 /(-2 \varepsilon)$ in the one-loop amplitudes of the soft function [13]. As noted above, we use the scheme of Ref. [8], in which the soft function is the full eikonal amplitude divided by the low-mass limit of the square root of the form factor for each external eikonal line. The one-loop soft anomalous dimension matrices found from the $\mathcal{M}_{i j}^{(1)}$ are then given by

$$
\begin{aligned}
\boldsymbol{\Gamma}_{S}^{(1)} & =-\frac{1}{2} \sum_{j} \sum_{i \neq j}\left(\mathbf{T}_{i} \cdot \mathbf{T}_{j}\right) I^{(-1)}\left(p_{i}, p_{j}\right)-\frac{1}{2} \sum_{j} C_{j} \ln \left(\frac{\mu^{2}}{m_{j}^{2}}\right) \\
& =-\frac{1}{2} \sum_{j} \sum_{i \neq j}\left(\mathbf{T}_{i} \cdot \mathbf{T}_{j}\right)\left[I^{(-1)}\left(p_{i}, p_{j}\right)-\ln \left(\frac{\mu^{2}}{m_{i} m_{j}}\right)\right]
\end{aligned}
$$

where in the first line we subtract for each external eikonal a term that is collinear-singular in the massless limit, and where $C_{q}=C_{\bar{q}}=C_{F}, C_{g}=C_{A}$ refer to the color content of the eikonal lines. This procedure eliminates all collinear logarithms, as may be readily verified from the expressions for $I^{(-1)}$ given in the appendix. To verify the second line of Eq. (4), we recall the color identities, [17]

$$
\sum_{i} \mathbf{T}_{i}=0, \quad \mathbf{T}_{j} \cdot \mathbf{T}_{j}=C_{j}
$$

with $i=F, A$. We are now ready for an analysis of the double-exchange diagrams.

The coefficients of $\left(\alpha_{s} / \pi\right)^{2}$ in the two-loop exchange diagrams of Fig. 1 1 , involving lines $i, j$ and $k$, can be written in terms of the coordinate space integrals, $I$, Eq. (3), as

$$
\mathcal{M}^{(2)}\left(p_{i}, p_{j}, p_{k}, \varepsilon\right)=\left.c^{2}(\varepsilon) m_{j}^{4 \varepsilon}\left(\mathbf{T}_{i} \cdot \mathbf{T}_{j}\right)\left(\mathbf{T}_{j} \cdot \mathbf{T}_{k}\right) \int_{0}^{\infty} \frac{d \lambda_{j}^{\prime}}{\lambda_{j}^{\prime 1-2 \varepsilon}} I\left(p_{i}, p_{j}, \varepsilon\right) \int_{0}^{\lambda_{j}^{\prime}} \frac{d \lambda_{j}}{\lambda_{j}^{1-2 \varepsilon}} I\left(p_{k}, p_{j}, \varepsilon\right)\right|_{U V},
$$

where the variables $\lambda_{j}$ and $\lambda_{j}^{\prime}$ represent the locations of the vertices along eikonal line $j$, to which two gluons are attached, as in Fig. 1. The ordered pairs of arguments $p_{i}, p_{j}\left(p_{j}, p_{k}\right)$ denote the eikonal lines connected by the outer (inner) gluon exchange of the diagram. The double pole of Eq. (6) is associated with the independent limits $\lambda_{j} \rightarrow 0$ and $\lambda_{j}^{\prime} \rightarrow 0$ of distances from the origin to the vertices along the $j$ eikonal. The factors $I\left(p_{i}, p_{j}, \epsilon\right)$ and $I\left(p_{k}, p_{j}, \epsilon\right)$ are the result of integrals like Eq. (3), which are over the ratios of origin-to-vertex distances. These integrals converge at both zero and infinity for fixed masses and momentum invariants. Thus, the individual poles arise from independent scaling of the two vertices of the $i j$ - or $k j$-exchange in Fig. 1 to the origin. Any remaining single pole represents a scaling of the four vertices of the entire diagram together to the origin. The latter defines the diagram's contribution to the two-loop anomalous dimension.

The UV poles of the $\lambda_{j}$ integrals in Eq. (6) can readily be isolated, because the functions $I\left(p_{i}, p_{j}, \varepsilon\right)$, defined in Eq. (3), are independent of the $\lambda_{j}$ 's. We find,

$$
\begin{aligned}
\mathcal{M}^{(2)}\left(p_{i}, p_{j}, p_{k}, \varepsilon\right)=c^{2}(\varepsilon) m_{j}^{4 \varepsilon}\left(\mathbf{T}_{i} \cdot \mathbf{T}_{j}\right) & \left(\mathbf{T}_{j} \cdot \mathbf{T}_{k}\right)\left[\frac{1}{8 \varepsilon^{2}} I^{(-1)}\left(p_{i}, p_{j}\right) I^{(-1)}\left(p_{k}, p_{j}\right)\right. \\
+ & \left.\frac{1}{8 \varepsilon} I^{(-1)}\left(p_{i}, p_{j}\right) I^{(0)}\left(p_{k}, p_{j}\right)+\frac{1}{8 \varepsilon} I^{(0)}\left(p_{i}, p_{j}\right) I^{(-1)}\left(p_{k}, p_{j}\right)\right]
\end{aligned}
$$

with both double and single poles, in a form that is clearly symmetric in the pairs $i j$ and $k j$ [4].

The complete set of two-loop diagrams corresponding to Fig. 1 includes as well counterterms for the UV-divergent subdiagram consisting of the (inner) exchange as shown in Fig. 3. For Fig. [3a, this one-loop counterterm is simply 
$-\mathcal{M}^{(1)}\left(p_{k}, p_{j}, \varepsilon\right)$, defined by Eq. (2). For convenience, we include the factor $c(\varepsilon)$ as part of the renormalization scheme. The diagrammatic representation of the corresponding two-loop contribution is shown in Fig. 3 a, and is given by

$$
\begin{gathered}
\mathcal{M}^{(2, c t r)}\left(p_{i}, p_{j}, p_{k}, \varepsilon\right)=-\left.c^{2}(\varepsilon) m_{j}^{4 \varepsilon}\left(\mathbf{T}_{i} \cdot \mathbf{T}_{j}\right)\left(\mathbf{T}_{j} \cdot \mathbf{T}_{k}\right) \int_{0}^{\infty} \frac{d \lambda_{j}^{\prime}}{\lambda_{j}^{\prime 1-2 \varepsilon}} I\left(p_{i}, p_{j}, \varepsilon\right) \frac{1}{2 \varepsilon} I^{(-1)}\left(p_{k}, p_{j}\right)\right|_{U V} \\
=-c^{2}(\varepsilon) m_{j}^{4 \varepsilon}\left(\mathbf{T}_{i} \cdot \mathbf{T}_{j}\right)\left(\mathbf{T}_{j} \cdot \mathbf{T}_{k}\right)\left[\left(\frac{1}{2 \varepsilon}\right)^{2} I^{(-1)}\left(p_{i}, p_{j}\right) I^{(-1)}\left(p_{k}, p_{j}\right)+\frac{1}{4 \varepsilon} I^{(0)}\left(p_{i}, p_{j}\right) I^{(-1)}\left(p_{k}, p_{j}\right)\right]
\end{gathered}
$$

again providing both single- and double-pole contributions. Combining Fig. 1] with its one-loop counterterm, Fig. $[3$, we find

$$
\begin{array}{r}
\mathcal{M}^{(2)}\left(p_{i}, p_{j}, p_{k}, \varepsilon\right)+\mathcal{M}^{(2, c t r)}\left(p_{i}, p_{j}, p_{k}, \varepsilon\right)=c^{2}(\varepsilon) m_{j}^{4 \varepsilon}\left(\mathbf{T}_{i} \cdot \mathbf{T}_{j}\right)\left(\mathbf{T}_{j} \cdot \mathbf{T}_{k}\right)\left(-\frac{1}{8 \varepsilon^{2}} I^{(-1)}\left(p_{i}, p_{j}\right) I^{(-1)}\left(p_{k}, p_{j}\right)\right. \\
\left.-\frac{1}{8 \varepsilon}\left[I^{(0)}\left(p_{i}, p_{j}\right) I^{(-1)}\left(p_{k}, p_{j}\right)-I^{(-1)}\left(p_{i}, p_{j}\right) I^{(0)}\left(p_{k}, p_{j}\right)\right]+\mathcal{O}\left(\varepsilon^{2}\right)\right)
\end{array}
$$

In this expression, we see directly that the double pole is symmetric in the momentum pairs $p_{i}, p_{j}$ and $p_{k}, p_{j}$, while the single pole is purely antisymmetric.

We next combine the foregoing result with the diagram in which the roles of the pairs $i j$ and $j k$ are exchanged, as in Figs. 1b and 3b. Including color factors, we find the following pole and color structure,

$$
\begin{array}{r}
\mathcal{M}^{(2)}\left(p_{i}, p_{j}, p_{k}, \varepsilon\right)+\mathcal{M}^{(2, c t r)}\left(p_{i}, p_{j}, p_{k}, \varepsilon\right)+(i \leftrightarrow k)=-\left\{\mathbf{T}_{i} \cdot \mathbf{T}_{j}, \mathbf{T}_{j} \cdot \mathbf{T}_{k}\right\} \frac{c^{2}(\varepsilon) m_{j}^{4 \varepsilon}}{2(2 \varepsilon)^{2}} I^{(-1)}\left(p_{i}, p_{j}\right) I^{(-1)}\left(p_{k}, p_{j}\right) \\
+\left[\mathbf{T}_{i} \cdot \mathbf{T}_{j}, \mathbf{T}_{j} \cdot \mathbf{T}_{k}\right] \frac{c^{2}(\varepsilon) m_{j}^{4 \varepsilon}}{8 \varepsilon}\left[I^{(-1)}\left(p_{i}, p_{j}\right) I^{(0)}\left(p_{k}, p_{j}\right)-I^{(0)}\left(p_{i}, p_{j}\right) I^{(-1)}\left(p_{k}, p_{j}\right)\right] \cdot(10)
\end{array}
$$

We recognize that the single pole terms in this expression enter entirely with antisymmetric color factors, while the double poles are entirely symmetric in color [4].

The combination of all such three-eikonal double exchanges (DE) can now be written down for an arbitrary number of eikonal lines. The double pole terms of Eq. (10) are cancelled by double poles of two-loop counterterms, which we see are the expansion of exponentiated one-loop counterterms. This leaves the single-pole, color-antisymmetric terms. Recalling [13] that the contributions of these diagrams to the two-loop anomalous dimension matrix is the residue of the single pole, $-1 /(4 \varepsilon)$, we find

$$
\boldsymbol{\Gamma}_{S}^{(3 E, \mathrm{DE})}=\left(\frac{\alpha_{s}}{\pi}\right)^{2} \frac{1}{2} \sum_{i>j>k=1}^{n} i f^{a b c} \mathbf{T}_{i}^{a} \mathbf{T}_{j}^{b} \mathbf{T}_{k}^{c} \sum_{I, J, K=(i, j, k)} \varepsilon^{I J K} I^{(-1)}\left(p_{I}, p_{J}\right) I^{(0)}\left(p_{K}, p_{J}\right)
$$

where we have exhibited the antisymmetric color structure explicitly by carrying out the commutators in Eq. (10). In this sum, the $\varepsilon$-expansions of the overall factors Eq. (10), $c^{2}(\varepsilon) m_{j}^{4 \varepsilon}$, cancel because of the antisymmetry in (11), which is invariant under any modification $I^{(0)}\left(p_{i}, p_{j}\right) \rightarrow I^{(0)}\left(p_{i}, p_{j}\right)+$ const $\times I^{(-1)}\left(p_{i}, p_{j}\right)$. In fact, we can generalize this result to any overall rescaling of the integrals $I$,

$$
I\left(p_{i}, p_{j}, \varepsilon\right) \rightarrow C\left(p_{i}, p_{j}, \varepsilon\right) I\left(p_{i}, p_{j}, \varepsilon\right),
$$

where $C$ has an expansion, $C\left(p_{i}, p_{j}, \varepsilon\right)=1+\varepsilon C^{(1)}\left(p_{i}, p_{j}\right)+\ldots$ As can be seen immediately from Eq. (10), any such overall rescaling has no effect on the antisymmetric single pole terms of the two-loop anomalous dimension. Such a rescaling, of course, does change the symmetric color terms at the single-pole level, and should be thought of as a change in scheme for the soft function. Equation (11) is in the form found in Ref. [6]. The exact expressions for the integrals $I\left(p_{i}, p_{j}, \varepsilon\right)$, in terms of which our results have been expressed so far, are given explicitly in the appendix for both space- and time-like kinematics. These results confirm the consistency of the two approaches in Refs. [4] and [6] that we set out to establish.

To conclude this section, we note for completeness the form of the full three-eikonal anomalous dimension matrix, which is found by combining the results from the double-exchange diagrams, Eq. (11), with the three-gluon diagram, Fig. 2. The latter has the same color structure, and can be incorporated into Eq. (11) by simply adding terms to the factors $I^{(0)}$ in Eq. (11) [6],

$$
\boldsymbol{\Gamma}_{S}^{(3 E)}=\left(\frac{\alpha_{s}}{\pi}\right)^{2} \frac{1}{2} \sum_{i>j>k=1}^{n} i f^{a b c} \mathbf{T}_{i}^{a} \mathbf{T}_{j}^{b} \mathbf{T}_{k}^{c} \sum_{I, J, K=(i, j, k)} \varepsilon^{I J K} I^{(-1)}\left(p_{I}, p_{J}\right)\left[I^{(0)}\left(p_{K}, p_{J}\right)+I_{3 g}\left(p_{K}, p_{J}\right)\right]
$$


The explicit result for $I_{3 g}$ can be found in the appendix. Finally, we note that to construct the full two-loop anomalous dimension matrix for an $n$-point amplitude one needs to combine Eq. (13) with the diagrams in which gluons connect to only two eikonals. The latter, which have the same color structure as the one-loop matrix, have been given explicitly in Ref. [8].

\section{MASSLESS EIKONALS IN COORDINATE SPACE}

The diagrams treated in the previous section, of course, also appear in the calculation of the anomalous dimension matrix with massless eikonal lines [18]. In Ref. [13], the same double exchange diagrams considered above, Figs. 1a,b, were shown for the massless case to give vanishing contributions to the two-loop anomalous dimension matrix using a momentum-space analysis. That is, their only contributions are cancelled by two-loop counterterms that are expansions of exponentiated one-loop counterterms. It is worthwhile examining how this result is maintained in coordinate space when taking into account the counterterm diagrams of Fig. 3 .

We begin by recalling that the soft function for massless lines must be defined to eliminate collinear poles on a loop-by-loop basis. We choose the form employed in Ref. [13], and mentioned above, where the soft function is defined as the ratio of the full eikonal amplitude to the product of the square roots of color-singlet form factors, one for each eikonal line. This is the analog of the color diagonal subtraction in Eq. (4) in the massive case. We will not need the details of this construction here, but observe that it requires that at each loop order, double poles appear with only color-diagonal coefficients after the sum over diagrams. We will see this feature emerge explicitly below, as we discuss the color structure of one- and two-loop exchange diagrams, beginning with the massless analog of the one-loop diagram, Eq. (2).

For computations involving massless eikonals, we continue to use momenta $p_{i}$ in place of dimensionless fourvelocities. Because the integrands that define the amplitudes are scaleless in the velocities, this is a trivial substitution before integration. For $p_{i}^{2}=p_{j}^{2}=0$ we then have

$$
\begin{aligned}
\mu^{2 \varepsilon} \mathcal{M}^{(1)}\left(p_{i} \cdot p_{j}, \varepsilon\right)_{p_{i}^{2}=p_{j}^{2}=0} & =\left.\mu^{2 \varepsilon} c(\varepsilon)\left(\mathbf{T}_{i} \cdot \mathbf{T}_{j}\right) \int_{0}^{\infty} d \lambda_{j} \int_{0}^{\infty} d \lambda_{i} \frac{p_{i} \cdot p_{j}}{\left[\left(\lambda_{j} p_{j}-\lambda_{i} p_{i}\right)^{2}-i \epsilon\right]^{1-\varepsilon}}\right|_{U V} \\
& =\left.\mu^{2 \varepsilon} c(\varepsilon)\left(\mathbf{T}_{i} \cdot \mathbf{T}_{j}\right) \int_{0}^{\infty} d \lambda_{j} \int_{0}^{\infty} d \lambda_{i} \frac{p_{i} \cdot p_{j}}{\left[-2 p_{i} \cdot p_{j} \lambda_{i} \lambda_{j}-i \epsilon\right]^{1-\varepsilon}}\right|_{U V},
\end{aligned}
$$

where we have multiplied by $\mu^{2 \varepsilon}$ so that both sides of this expression are dimensionless. This will help motivate our choice of renormalization scheme for this integral. As in our discussion of the massive case, we treat $-2 p_{i} \cdot p_{j}$ as a positive quantity, and then analytically continue to negative values. The coordinate space expression in (14) has two scaleless integrals rather than one, as in Eq. (2). The treatment of these integrals requires some discussion, even in the one-loop case.

The presence of mixed infrared and collinear singularities in a Lorentz-invariant integral make it impossible to maintain at the same time the correct power behavior under the scaling of momenta and invariance under rescalings of the velocities, a feature sometimes referred to as the "cusp anomaly" [12]. As a result, the prescription to identify the ultraviolet pole does not, as in the massive case, uniquely fix the coefficient of that pole. We can organize these ambiguities by changing variables from the $\lambda$ 's, which have dimensions of length squared, to dimensionless variables. This is a unique change of variables in this case, if we insist that the integral (14) depend only on $p_{i} \cdot p_{j}$. We are therefore led to the dimensionless variables

$$
l_{j} \equiv \lambda_{j}\left(-2 p_{i} \cdot p_{j}\right), \quad l_{i} \equiv \lambda_{i}\left(-2 p_{i} \cdot p_{j}\right) .
$$

After this change of variables, the dimensional content of the integral is manifest. To isolate the ultraviolet pole, we use the symmetry between $l_{i}$ and $l_{j}$, and write

$$
\mu^{2 \varepsilon} \mathcal{M}^{(1)}\left(p_{i} \cdot p_{j}, \varepsilon\right)_{p_{i}^{2}=p_{j}^{2}=0}=-\left.\left(\mathbf{T}_{i} \cdot \mathbf{T}_{j}\right) c(\varepsilon)\left(\frac{\mu^{2}}{-2 p_{i} \cdot p_{j}}\right)^{\varepsilon} \int_{0}^{\infty} \frac{d l_{j}}{l_{j}^{1-\varepsilon}} \int_{l_{j}}^{\infty} \frac{d l_{i}}{l_{i}^{1-\varepsilon}}\right|_{U V} .
$$

The $l_{i}$ integral is carried out at fixed $l_{j}$ for infrared regularization $(\varepsilon<0)$, to give a simple pole. Next, the ultraviolet pole of the $l_{j}$ integral is isolated. This results, of course, in an expression with both single and double poles,

$$
\begin{aligned}
\mu^{2 \varepsilon} \mathcal{M}^{(1)}\left(p_{i} \cdot p_{j}, \varepsilon\right)_{p_{i}^{2}=p_{j}^{2}=0} & =\left(\mathbf{T}_{i} \cdot \mathbf{T}_{j}\right) c(\varepsilon)\left(\frac{\mu^{2}}{-2 p_{i} \cdot p_{j}}\right)^{\varepsilon} \frac{1}{2 \varepsilon^{2}} \\
& =\frac{1}{2}\left(\mathbf{T}_{i} \cdot \mathbf{T}_{j}\right)\left(\frac{1}{\varepsilon^{2}}-\frac{1}{\varepsilon} \ln \left(\frac{-2 p_{i} \cdot p_{j}}{\mu^{2}}\right)\right)+\ldots
\end{aligned}
$$


Momentum-dependence appears only in single poles, while double poles are momentum-independent. In either form of (17), we can revert to a velocity dependence by choosing $\beta_{i} \equiv p_{i} / \mu$. Keeping the momentum-dependence, we can identify the first line of Eq. (17) as the eikonal contribution to the familiar function $\mathbf{I}^{(1)}$ defined in [19]. As in the massive case above, the choice between the first and second lines is a choice of renormalization scheme. In fact, we could use the first line to define an alternative scheme to define the soft function. In this discussion, however, we stick with the choice of pure poles, as in Ref. [13].

As in the example of Eq. (4), color conservation, Eq. (5), implies a form in which, as anticipated, the double poles organize themselves into color-diagonal terms,

$$
\mu^{2 \varepsilon} \sum_{j \neq i} \mathcal{M}^{(1)}\left(p_{i} \cdot p_{j}, \varepsilon\right)_{p_{i}^{2}=p_{j}^{2}=0}=-\left(\frac{1}{2 \varepsilon}\left(\sum_{j \neq i} \mathbf{T}_{i} \cdot \mathbf{T}_{j}\right) \ln \left(\frac{-2 p_{i} \cdot p_{j}}{\mu^{2}}\right)+\frac{1}{4 \varepsilon^{2}} C_{i}\right) .
$$

The order $\alpha_{s}$ double pole terms cancel against the form factors in the definition of the soft function. The resulting one-loop counterterms that correspond to Eq. (18) are then pure single poles.

Going on to two loops, we follow the reasoning leading to Eq. (11) in the massive case. The essential difference is that all integrals remain scaleless, and we do not encounter convergent integrals like Eq. (3), whose expansion in $\varepsilon$ can give single poles at two loops. For the massless case, the two-loop integrals produce no expansion in $\varepsilon$ beyond the pole terms that define the scheme. Alternately, there is no pole associated with a single scaling of all the vertices in the diagram together, of the sort we identified in Eq. (6) with massive eikonals. That is, in the massless case each integral of the two-loop diagram is fully independent. The massless analog of Eq. (6) for Fig. 10 is then,

$$
\begin{aligned}
\mu^{4 \varepsilon} \mathcal{M}^{(2)}\left(p_{i} \cdot p_{j}, p_{k} \cdot p_{j}, \varepsilon\right)= & \mu^{4 \varepsilon} c^{2}(\varepsilon)\left(\mathbf{T}_{k} \cdot \mathbf{T}_{j}\right)\left(\mathbf{T}_{i} \cdot \mathbf{T}_{j}\right) \int_{0}^{\infty} d \lambda_{j}^{\prime} \int_{0}^{\infty} d \lambda_{k} \frac{p_{k} \cdot p_{j}}{\left[-2 p_{k} \cdot p_{j} \lambda_{k} \lambda_{j}^{\prime}-i \epsilon\right]^{1-\varepsilon}} \\
& \times\left.\int_{0}^{\lambda_{j}^{\prime}} d \lambda_{j} \int_{0}^{\infty} d \lambda_{i} \frac{p_{i} \cdot p_{j}}{\left[-2 p_{i} \cdot p_{j} \lambda_{i} \lambda_{j}-i \epsilon\right]^{1-\varepsilon}}\right|_{U V},
\end{aligned}
$$

where we assume all lightlike momenta, and where the ordering of the arguments of $\mathcal{M}^{(2)}$ determines the ordering of the color matrices. The integrand here is manifestly symmetric in $p_{i}$ and $p_{k}$. As a result, Fig. 1a differs from Fig. 1b only by the orderings of their integration parameters $\lambda_{j}$ and $\lambda_{j}^{\prime}$ and of their color factors. A simple exchange of the two integration labels eliminates the antisymmetric part in the sum of the two diagrams, and we find

$$
\mu^{4 \varepsilon}\left[\mathcal{M}^{(2)}\left(p_{i} \cdot p_{j}, p_{k} \cdot p_{j}, \varepsilon\right)+\mathcal{M}^{(2)}\left(p_{k} \cdot p_{j}, p_{i} \cdot p_{j}, \varepsilon\right)\right]=\frac{1}{2}\left\{\mu^{2 \varepsilon} \mathcal{M}^{(1)}\left(p_{i} \cdot p_{j}, \varepsilon\right), \mu^{2 \varepsilon} \mathcal{M}^{(1)}\left(p_{k} \cdot p_{j}, \varepsilon\right)\right\} .
$$

Only the symmetric part survives in the sum of the two diagrams, considered as color matrices. This is the coordinate space analog of a momentum space argument leading to the same result in Ref. [13]. As in the massive case, we are left to analyze potentially antisymmetric contributions from diagrams like Figs. 3 , with counterterms. Unlike the massive case, however, the one-loop integrals of these diagrams are scaleless in both integration variables, and there are no finite terms, beyond those that may have been included in the scheme that defines the one-loop counterterms. This ensures that the counterterm diagrams are proportional to the original diagrams, and that their sum remains symmetric. In principle, these one-loop counterterms include double as well as single poles, but as we have seen in our one-loop example, double poles systematically cancel in the soft anomalous dimension matrix, a result that extends to all orders [1, 20].

The origin of the difference between the massive and massless cases is easy to trace to the one-loop integrals of Eqs. (31) and (16), respectively. The integrands in the two cases differ only when the scaled integration variable $\sigma=\lambda_{i} / \lambda_{j}$ in (3) is large (or small) enough so that

$$
\frac{\lambda_{i}}{\lambda_{j}} \geq \frac{2 p_{i} \cdot p_{j}}{p_{i}^{2}} \quad \text { or } \quad \frac{\lambda_{j}}{\lambda_{i}} \geq \frac{2 p_{i} \cdot p_{j}}{p_{j}^{2}} .
$$

Only for these "collinear" regions do the integrands distinguish between massless and massive eikonal lines. For any fixed mass, however, these regions are always present. Within each diagram, therefore, the limit of zero mass does not commute with the integrations. This is not the case for the full soft function, however, because these collinear regions, whether regularized by dimensions or masses, cancel in the ratio of the full eikonal amplitude to jet functions $[1,[15,20]$, after the sum over a gauge-invariant set of diagrams. 


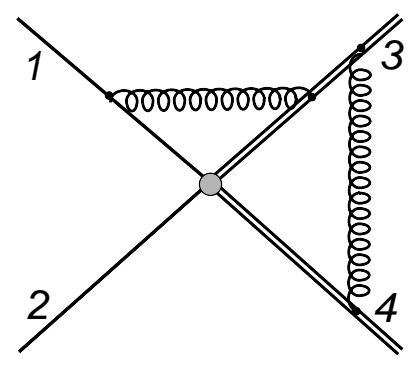

(a)

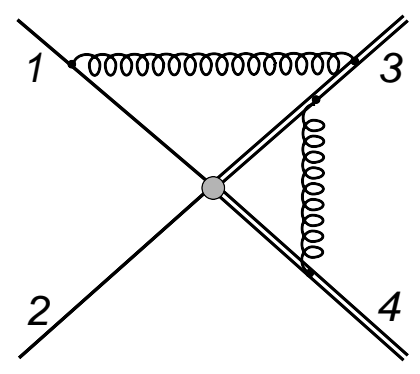

(c)

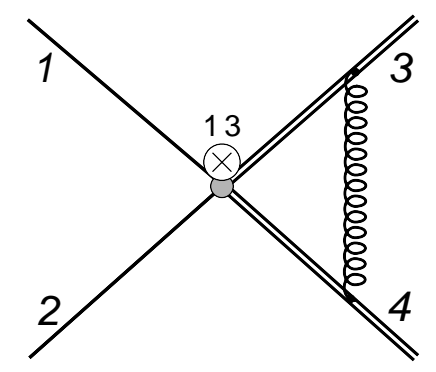

(b)

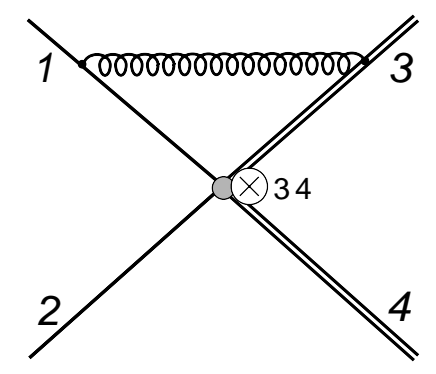

(d)

FIG. 5: Two-loop double-exchange diagrams and their one-loop counterterms at order $g^{4}$ in the two-to-two scattering reactions $q \bar{q} \rightarrow Q \bar{Q}$ and $g g \rightarrow Q \bar{Q}$.

\section{ANOMALOUS DIMENSIONS NEAR ABSOLUTE THRESHOLD}

In this section, we discuss the interpretation of threshold behavior of the eikonal soft function representing a twoto-two reaction of the type $q \bar{q} \rightarrow Q \bar{Q}$ or $g g \rightarrow Q \bar{Q}$, as in Fig. [5. We will concentrate on the effects of the double exchange diagrams shown there, involving (for example) an incoming light quark or gluon, labelled 1, and the two outgoing particles, labelled 3 and 4 . Our goal here is to rederive and interpret the non-uniform behavior in the massive anomalous dimension matrix found in Ref. [6] at absolute threshold, where $s \rightarrow 4 M^{2}$, with $M$ the heavy quark mass. We will confirm and interpret the conclusion [8] that this behavior relates to a region in momentum space in which the eikonal approximation does not apply to the underlying partonic cross section.

Equation (11) above shows how the double-exchange diagrams contribute to the anomalous dimension matrix. For

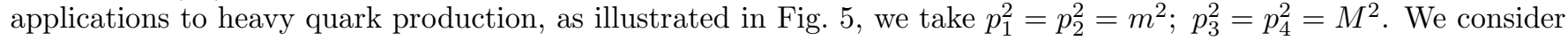
the incoming mass $m$ to be small, $m<<M$, and with heavy quark production in mind will eventually take it to zero. To find the threshold, $s=\left(p_{1}+p_{2}\right)^{2} \rightarrow 4 M^{2}$, behavior for this two-to-two process, we take the $\beta=\sqrt{1-4 M^{2} / s} \rightarrow 0$, with $\beta$ the center of mass velocity for the heavy pair. ${ }^{3}$ Specifically, using the expressions given in the appendix for $m \ll M$ we find for the terms in Eq. (11) with $i=4, j=3, k=1$ corresponding to Fig. 5 .

$$
\begin{aligned}
I^{(0)}\left(p_{4}, p_{3}\right) I^{(-1)}\left(p_{1}, p_{3}\right)-I^{(-1)}\left(p_{4}, p_{3}\right) I^{(0)}\left(p_{1}, p_{3}\right) & =\left(1-\frac{i \pi}{2 \beta}\right)\left(\ln ^{2}\left(\frac{m M}{-t_{1}}\right)+\frac{\pi^{2}}{6}\right) \\
& +\ln \left(\frac{m M}{-t_{1}}\right)\left[\frac{1}{\beta}\left(-\frac{\pi^{2}}{2}-i \pi \ln (4 \beta)\right)+2\right]+\mathcal{O}(\beta),
\end{aligned}
$$

where $t_{1}=-2\left(p_{1} \cdot p_{3}\right)$ and $u_{1}=-2\left(p_{1} \cdot p_{4}\right)$ are customary invariants.

As noted in Sec. III above, the double logarithm in Eq. (22), which originates from the collinear region, must be cancelled by the diagram with a three-gluon interaction [6], which we reproduce in the appendix, Eq. (A12). After this cancellation, in the threshold limit the diagrams of Fig. 5 then give a contribution to the anomalous dimension matrix,

$$
\boldsymbol{\Gamma}_{S}^{(431, \text { figs })}=\frac{1}{2} i f^{a b c} \mathbf{T}_{4}^{a} \mathbf{T}_{3}^{b} \mathbf{T}_{1}^{c}\left\{\ln \left(\frac{m M}{-t_{1}}\right)\left[\frac{1}{\beta}\left(-\frac{\pi^{2}}{2}-i \pi \ln (4 \beta)\right)+2\right]+\left(1-\frac{i \pi}{2 \beta}\right) \frac{\pi^{2}}{6}\right\}+\mathcal{O}(\beta) .
$$

\footnotetext{
${ }^{3}$ In terms of the formulas given in the appendix, this corresponds to $x_{34} \rightarrow 1$ (equivalently, $v_{34} \rightarrow 0$ ) in Eqs. A99 and A11) and $x_{13} \rightarrow 0$ (equivalently, $v_{13} \rightarrow 1$ ) in Eqs. A3 and A4.
} 
This result is still infrared divergent in the limit $m \rightarrow 0$. When we add the diagrams in which the roles of lines 3 and 4 are reversed, however, we find the same infrared-finite $\boldsymbol{\Gamma}_{S}$ given in Eqs. (53)-(55) of Ref. [6],

$$
\boldsymbol{\Gamma}_{S}^{(431)}=\frac{1}{2} i f^{a b c} \mathbf{T}_{4}^{a} \mathbf{T}_{3}^{b} \mathbf{T}_{1}^{c} \ln \left(\frac{u_{1}}{t_{1}}\right)\left[\frac{1}{\beta}\left(-\frac{\pi^{2}}{2}-i \pi \ln (4 \beta)\right)+2\right]+\mathcal{O}(\beta) .
$$

Equation (24) has a very interesting behavior in the limit $\beta \rightarrow 0$. By adopting the center of mass relation, $t_{1}=$ $-s(1-\beta \cos \theta) / 2$ and similarly for $u_{1}$, we observe that

$$
\boldsymbol{\Gamma}_{S}^{(431)}=-i f^{a b c} \mathbf{T}_{4}^{a} \mathbf{T}_{3}^{b} \mathbf{T}_{1}^{c} \cos \theta\left(\frac{\pi^{2}}{2}+i \pi \ln (4 \beta)\right)+\mathcal{O}(\beta)
$$

in which the Coulomb singularity combines with an angular-dependent factor that vanishes at threshold to give a term that remains finite for $\beta \rightarrow 0$. As pointed out in Ref. [6], this term depends on the center of mass scattering angle, no matter how small $\beta$ is. The $\beta \rightarrow 0$ limit would thus appear to be ambiguous. What are we to make of this?

The factorization formalism that leads to the soft anomalous dimension matrix [1, 20] applies in the approximation that the momentum of the active partons is much larger than that of the soft radiation which the anomalous dimension matrix is used to resum. The use of an anomalous dimension matrix thus requires that the eikonal approximation apply, that is, that the emission or absorption of this radiation leaves the four-velocities of the heavy quarks essentially unchanged. In fact, in the momentum region that produces the Coulomb singularity, the center of mass kinetic energy of the quark pair is order $m \beta^{2}$ in virtual as well as real states. Soft radiation emitted by the produced pair can carry an energy of no more than this order without violating the eikonal approximation and washing out the $1 / \beta$ dependence. Thus, as $\beta$ vanishes, the energy range of virtual or real radiation to which we can apply the eikonal approximation vanishes even faster, as the square of the relative velocity. Although the $\beta$-independent piece of the anomalous dimension in (25), which couples singlet and octet color states, is present at any finite value of $\beta$, its range of applicability shrinks to zero for $\beta \rightarrow 0$. Correspondingly, as argued in Ref. [8], in the inclusive cross section, as opposed to an elastic amplitude, it is only the range of energies $m \geq \omega \geq m \beta^{2}$ that contribute to threshold logarithms.

The Coulomb singularity at zero relative velocity $\beta$ is a characteristic feature of heavy particle production and the analysis of bound-state formation [21]. We can trace the origin of the $1 / \beta$ dependence in a particularly simple fashion for the integrals $I\left(p_{i}, p_{j}, \varepsilon\right)$, defined in Eq. (3) for the exchange diagrams. Setting for definiteness $j=3, k=4$, the integration variable $\sigma=\lambda_{3} / \lambda_{4}$ measures the relative distances from the origin along the two heavy-particle eikonal lines, with momenta $p_{3}$ and $p_{4}$. The $\sigma$ integration contour encounters two singularities, at

$$
\begin{aligned}
\sigma_{ \pm} & =\frac{p_{3} \cdot p_{4}}{m^{2}} \pm \frac{1}{m^{2}} \sqrt{\left(p_{3} \cdot p_{4}\right)^{2}-m^{4}} \pm i \epsilon \\
& =1 \pm 2 \beta \pm i \epsilon+\mathcal{O}\left(\beta^{2}\right)
\end{aligned}
$$

In the limit of vanishing relative velocity, then, the $\sigma$ integral is pinched between coalescing singularities at $\sigma=1$, whose separation vanishes linearly with $\beta$. In terms of the original integrals over $\lambda_{3}$ and $\lambda_{4}$, the singularity at $\sigma=1 \pm 2 \beta$ corresponds to singularities in (say) $\lambda_{3}$ at $\lambda_{3}=\lambda_{4}(1 \pm 2 \beta)$. In particular, for $\beta=0$, the two ends of the gluon propagator are at the same point in coordinate space, $\lambda_{3} p_{3}=\lambda_{4} p_{4}$. We can use this analysis to interpret further the range of applicability of the anomalous dimension matrix.

Working in the $p_{3}, p_{4}$ center of mass frame, the quantities $\lambda_{3}+\lambda_{4}$ and $\lambda_{3}-\lambda_{4}$ are proportional to the temporal and spatial distances between two points along the $p_{3}$ and $p_{4}$ eikonals, and are conjugate to the energy and spatial momentum of emitted radiation. In the region that dominates the integrals, the conjugate of the spatial momenta is constrained to be smaller than the conjugate of the energy by a factor of $\beta$, corresponding to a range in spatial momentum larger than energy by a factor of $1 / \beta$. Thus, when the energy of gluon exchange approaches the scale of heavy quark kinetic energy, $m \beta^{2}$, the exchanged momentum approaches $m \beta$, which is the same order as the heavy quark momentum. In this region, the quarks can no longer by considered as recoilless sources, and the eikonal approximation is not reliable. In Ref. [7], an effective theory treatment for resummation in this region was developed, and in [8] an equivalent analysis in which the pair is replaced by a single Wilson line was employed. In either case, it is necessary to assume that radiation with energy-momentum scales up to $m \beta$ cancels in the sum over final states for the inclusive cross section.

Finally, it is interesting to note that the connection between $\cos \theta$ and the kinematic variables $s$ and $t_{1}$ used in the derivation of Eq. (25) is singular at absolute threshold. In two-to-two scattering processes, $s+t_{1}+u_{1}=0$, and the function $\Gamma_{S}^{(134)}$ in Eq. (24) is determined by two variables, which we can choose as $\left(s, t_{1}\right)$. The absolute threshold $\beta=0$ then represents the single point $\left(s=s_{0} \equiv 4 m^{2}, t_{1}=t_{0} \equiv-s_{0} / 2\right)$ in the physical region of the kinematical $\left(s, t_{1}\right)$ plane; see Fig. 6. The relationship between $t_{1}$ and $s$ can now be written as 


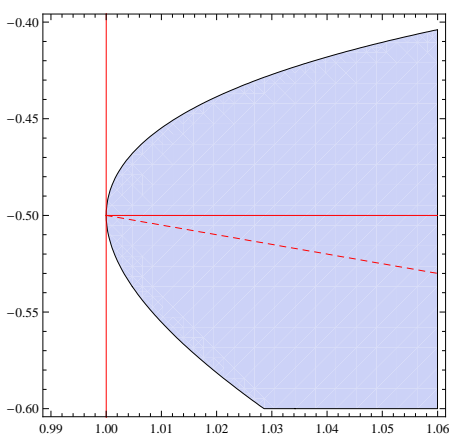

FIG. 6: Illustration of the physical region (shaded) in the $\left(s, t_{1}\right)$ kinematic plane. The normalization is chosen such that $s_{0}=1$. The solid lines depict the lines $\beta=0$ (i.e. $s=s_{0}$ ) and $t=t_{0}$. The dashed line denotes the set of points $t_{1}=-s / 2$ where the function $\Gamma_{S}^{(134)}$, defined in Eq. (24), vanishes due to antisymmetry (note that this line also crosses the absolute threshold point $\left.t_{0}=-s_{0} / 2\right)$.

$$
t_{1}=-\frac{s}{2}(1-\beta \cos \theta)=-\frac{s_{0}}{2}+\frac{\sqrt{s}}{2} \sqrt{s-s_{0}} \cos \theta-\frac{1}{2}\left(s-s_{0}\right) .
$$

At threshold, all derivatives of $t_{1}$ with respect to $s$ diverge unless $\cos \theta=0$. Therefore, in terms of figure 6 , all curves that approach absolute threshold at fixed, non-zero $\cos \theta$ converge to the vertical tangent to the boundary of the physical region at the point $t_{1}=t_{0}, s=s_{0}$. At the same time, it is straightforward to verify that along any straight line defined by the equation

$$
t_{1}=t_{0}+c\left(s-s_{0}\right), \quad u_{1}=t_{0}-(1+c)\left(s-s_{0}\right), \quad c<\infty
$$

the function $\Gamma_{S}^{(134)}$ in Eq. (24) vanishes at threshold and thus matches smoothly to the behavior of the function at $u_{1}=t_{1}[8]$. In a sense, as $\beta \rightarrow 0$, the cosine becomes ill-defined, because it no longer uniquely defines the Mandelstam variables. At the same time, for any fixed value of $\beta$, and in particular, for $\beta \rightarrow 1$, the full expression for the soft anomalous dimension as found in Ref. [6] applies without subtlety for gluon radiation in the range identified above.

\section{CONCLUSIONS}

In this note we have demonstrated the mutual consistency of a full position-space calculation of the massive soft anomalous dimension matrix following Ref. [4], and the momentum space results described in Ref. [6]. In particular, the color-symmetric structure of the two-loop double-exchange diagrams before renormalization shown in [4] is consistent with the results for planar diagrams found in Ref. [6], once renormalization is taken into account. We have also confirmed the consistency of results for the massive anomalous dimension matrix with those for the massless case and with the next-to-next-to leading logarithmic threshold resummations described in [7, 8]. Finally, we have seen how coordinate space analysis provides a clear interpretation of the non-uniform limit of the anomalous dimension matrix at absolute threshold.

\section{Acknowledgments}

A.M. would like to thank Michal Czakon, Kirill Melnikov and Shlomo Razamat for discussions. This work was supported in part by the National Science Foundation, grants PHY-0354776, PHY-0354822 and PHY-0653342. The work of A.M. is supported by a fellowship from the US LHC Theory Initiative through NSF grant 0705682.

\section{Appendix A: Explicit forms for the integrals}

The functions $I\left(p_{i}, p_{j}, \varepsilon\right)$, defined by Eq. (3), are one-dimensional integrals. For space-like kinematics (for example $p_{i}$ incoming, $p_{j}$ outgoing) the result in $d=4-2 \varepsilon$ dimensions and for arbitrary masses can be expressed as

$$
I\left(p_{i}, p_{j}, \varepsilon\right)=\frac{1}{1-2 \varepsilon}{ }_{2} F_{1}\left(\frac{1}{2}, 1 ; \frac{3}{2}-\varepsilon ; 1-\frac{m_{i}^{2} m_{j}^{2}}{\left(p_{i} \cdot p_{j}\right)^{2}}\right) .
$$


The above function has a well defined massless limit in $4-2 \varepsilon$ dimensions: ${ }_{2} F_{1}(1 / 2,1 ; 3 / 2-\varepsilon ; 1)=-(1-2 \varepsilon) /(2 \varepsilon)$. As can be seen from Eq. A1 all non-trivial dependence on the kinematical invariants and masses comes entirely through the combination:

$$
v_{i j}=\sqrt{1-\frac{m_{i}^{2} m_{j}^{2}}{\left(p_{i} \cdot p_{j}\right)^{2}}},
$$

familiar from the one-loop case [2]. Expressions for the functions $I^{(-1,0)}$ appearing in Eqs. (11), (13) can be obtained by expanding the hypergeometric function in $\varepsilon$, or by expansion of the integrand in Eq. (3) before integration. For expansion of the full expression (A1) we have used the program HypExp 22], as well as standard relations between the polylogarithmic functions. The resulting expressions can be written as

$$
\begin{aligned}
I^{(-1)}\left(p_{i}, p_{j}\right) & =-\frac{1}{2 v_{i j}} \ln \left(\frac{1-v_{i j}}{1+v_{i j}}\right), \\
I^{(0)}\left(p_{i}, p_{j}\right) & =\frac{1}{v_{i j}}\left\{-\operatorname{Li}_{2}\left(\frac{1-v_{i j}}{1+v_{i j}}\right)+\frac{1}{4} \ln ^{2}\left(\frac{1-v_{i j}}{1+v_{i j}}\right)+\ln \left(\frac{1-v_{i j}}{1+v_{i j}}\right) \ln \left(\frac{1+v_{i j}}{2 v_{i j}}\right)+\frac{\pi^{2}}{6}\right\} .
\end{aligned}
$$

The consistency of these results with those of Ref. [6] is readily checked by relating the variable $\beta_{i j}=-\cosh ^{-1}\left( \pm p_{i} \cdot\right.$ $\left.p_{j} / m_{i} m_{j}\right)$ to our $v_{i j}$ by

$$
\beta_{i j}=-\frac{1}{2} \ln \left(\frac{1-v_{i j}}{1+v_{i j}}\right), \quad \operatorname{coth} \beta_{i j}=\frac{1}{v_{i j}} .
$$

While compact, the expressions in Eqs. (A3) and (A4) are somewhat inconvenient for analytic continuation from space-like to time-like kinematics, because their momentum-dependence is only through the squares $\left(p_{i} \cdot p_{j}\right)^{2}$ in $v_{i j}$. For completeness and for use in our discussion of the threshold limit, we recall that analytic continuation is made straightforward by re-expressing the amplitudes in terms of the variable

$$
x_{i j}=\sqrt{\frac{1-v_{i j}}{1+v_{i j}}}=\frac{\sqrt{1-\frac{\left(m_{i}+m_{j}\right)^{2}}{s_{i j}}}-\sqrt{1-\frac{\left(m_{i}-m_{j}\right)^{2}}{s_{i j}}}}{\sqrt{1-\frac{\left(m_{i}+m_{j}\right)^{2}}{s_{i j}}}+\sqrt{1-\frac{\left(m_{i}-m_{j}\right)^{2}}{s_{i j}}}}, \quad v_{i j}=\frac{1-x_{i j}^{2}}{1+x_{i j}^{2}},
$$

with $s_{i j}=\left(p_{i}+p_{j}\right)^{2}$. The case of $m_{i}=m_{j}=m$ is of particular interest, where

$$
x_{i j}=\frac{\sqrt{1-\frac{4 m^{2}}{s_{i j}}}-1}{\sqrt{1-\frac{4 m^{2}}{s_{i j}}}+1}=-\frac{1-b_{i j}}{1+b_{i j}}, \quad \frac{m^{2}}{s_{i j}}=-\frac{x_{i j}}{\left(1-x_{i j}\right)^{2}},
$$

where $b_{i j}$ is the center-of-mass velocity for the pair $p_{i}, p_{j}$. For arbitrary masses, we have $x_{i j}>0$ for $s_{i j}<0$ (space-like) and $x_{i j}<0$ for $s_{i j}>0$ (time-like).

For any masses, we now rewrite the functions $I^{(-1,0)}$ in terms of the $x_{i j}$ as

$$
\begin{aligned}
I^{(-1)}\left(p_{i}, p_{j}\right) & =-\frac{1+x_{i j}^{2}}{1-x_{i j}^{2}} \ln \left(x_{i j}\right), \\
I^{(0)}\left(p_{i}, p_{j}\right) & =\frac{1+x_{i j}^{2}}{1-x_{i j}^{2}}\left(-\operatorname{Li}_{2}\left(x_{i j}^{2}\right)+\ln ^{2}\left(x_{i j}\right)-2 \ln \left(x_{i j}\right) \ln \left(1-x_{i j}^{2}\right)+\frac{\pi^{2}}{6}\right) .
\end{aligned}
$$

For space-like kinematics, where $1>x_{i j}>0$, these functions are real. Analytic continuation to time-like kinematics is found by the replacement: $x_{i j} \rightarrow-\left|x_{i j}\right|+i \varepsilon=\left|x_{i j}\right| e^{i \pi}$ (see Sec. 6 of Ref. [23] for details). Following this rule, the following terms should be added to Eqs. (A3), (A4) when the kinematics is time-like:

$$
\begin{aligned}
\Delta I^{(-1)}\left(p_{i}, p_{j}\right) & =-i \pi \frac{1+x_{i j}^{2}}{1-x_{i j}^{2}}=-\frac{i \pi}{v_{i j}} \\
\Delta I^{(0)}\left(p_{i}, p_{j}\right) & =\frac{1+x_{i j}^{2}}{1-x_{i j}^{2}}\left(-\pi^{2}+i \pi\left[2 \ln \left|x_{i j}\right|-2 \ln \left(1-x_{i j}^{2}\right)\right]\right) \\
& =-\frac{\pi^{2}}{v_{i j}}+\frac{i \pi}{v_{i j}}\left[\ln \left(\frac{1-v_{i j}}{1+v_{i j}}\right)+2 \ln \left(\frac{1+v_{i j}}{2 v_{i j}}\right)\right] .
\end{aligned}
$$


Finally, for space-like kinematics the result for the non-planar diagram in Fig. 2 as expressed in Eq. (13) above, reads $[6]$ :

$$
I_{3 g}\left(p_{i}, p_{j}\right)=-\ln ^{2}\left(x_{i j}\right)=-\frac{1}{4} \ln ^{2}\left(\frac{1-v_{i j}}{1+v_{i j}}\right)
$$

In time-like kinematics, it also receives the following term:

$$
\Delta I_{3 g}\left(p_{i}, p_{j}\right)=\pi^{2}-2 i \pi \ln \left(\left|x_{i j}\right|\right)=\pi^{2}-i \pi \ln \left(\frac{1-v_{i j}}{1+v_{i j}}\right) .
$$

[1] N. Kidonakis and G. Sterman, Nucl. Phys. B 505, 321 (1997) arXiv:hep-ph/9705234]; N. Kidonakis, G. Oderda and G. Sterman, Nucl. Phys. B 531, 365 (1998) arXiv:hep-ph/9803241.

[2] S. Catani, S. Dittmaier and Z. Trocsanyi, Phys. Lett. B 500, 149 (2001) arXiv:hep-ph/0011222.

[3] N. Kidonakis, Phys. Rev. Lett. 102, 232003 (2009) arXiv:0903.2561 [hep-ph]].

[4] A. Mitov, G. Sterman and I. Sung, Phys. Rev. D 79, 094015 (2009) [arXiv:0903.3241 [hep-ph]].

[5] T. Becher and M. Neubert, Phys. Rev. D 79, 125004 (2009) arXiv:0904.1021 [hep-ph]].

[6] A. Ferroglia, M. Neubert, B. D. Pecjak and L. L. Yang, arXiv:0907.4791 [hep-ph]; arXiv:0908.3676 [hep-ph].

[7] M. Beneke, P. Falgari and C. Schwinn, arXiv:0907.1443 [hep-ph].

[8] M. Czakon, A. Mitov and G. Sterman, Phys. Rev. D 80 (2009) 074017 arXiv:0907.1790 [hep-ph]].

[9] V. Ahrens, A. Ferroglia, M. Neubert, B. D. Pecjak and L. L. Yang, Phys. Lett. B 687, 331 (2010) arXiv:0912.3375 [hepph]]; V. Ahrens, A. Ferroglia, M. Neubert, B. D. Pecjak and L. L. Yang, arXiv:1003.5827 [hep-ph]; N. Kidonakis, Phys. Rev. D 81, 054028 (2010) arXiv:1001.5034 [hep-ph]].

[10] L. J. Dixon, L. Magnea and G. Sterman, JHEP 0808, 022 (2008) arXiv:0805.3515 [hep-ph]].

[11] T. Becher and M. Neubert, Phys. Rev. Lett. 102 (2009) 162001 arXiv:0901.0722 [hep-ph]]; JHEP 0906, 081 (2009) arXiv:0903.1126 [hep-ph]].

[12] E. Gardi and L. Magnea, JHEP 0903, 079 (2009) arXiv:0901.1091 [hep-ph]].

[13] S. M. Aybat, L. J. Dixon and G. Sterman, Phys. Rev. D 74, 074004 (2006) arXiv:hep-ph/0607309].

[14] G. Sterman and M. E. Tejeda-Yeomans, Phys. Lett. B 552, 48 (2003) arXiv:hep-ph/0210130.

[15] A. Mitov and S. Moch, JHEP 0705, 001 (2007) arXiv:hep-ph/0612149; T. Becher and K. Melnikov, JHEP 0706, 084 (2007) arXiv:0704.3582 [hep-ph]].

[16] J. Gluza, A. Mitov, S. Moch and T. Riemann, JHEP 0907, 001 (2009) [arXiv:0905.1137 [hep-ph]].

[17] S. Catani and M. H. Seymour, Phys. Lett. B 378, 287 (1996) hep-ph/9602277]; Nucl. Phys. B 485, 291 (1997) [Err.-ibid. B 510, 503 (1997)] hep-ph/9605323.

[18] I. A. Korchemskaya and G. P. Korchemsky, Phys. Lett. B 287, 169 (1992); G. P. Korchemsky and G. Marchesini, Nucl. Phys. B 406, 225 (1993) arXiv:hep-ph/9210281.

[19] S. Catani, Phys. Lett. B 427, 161 (1998) arXiv:hep-ph/9802439.

[20] A. Sen, Phys. Rev. D 28, 860 (1983).

[21] K. Hagiwara, Y. Sumino and H. Yokoya, Phys. Lett. B 666, 71 (2008) arXiv:0804.1014 [hep-ph]]; Y. Kiyo, J. H. Kuhn, S. Moch, M. Steinhauser and P. Uwer, Eur. Phys. J. C 60, 375 (2009) arXiv:0812.0919 [hep-ph]]; M. Beneke, M. Czakon, P. Falgari, A. Mitov and C. Schwinn, arXiv:0911.5166 [hep-ph].

[22] T. Huber and D. Maitre, Comput. Phys. Commun. 175, 122 (2006) arXiv:hep-ph/0507094; T. Huber and D. Maitre, Comput. Phys. Commun. 178, 755 (2008) arXiv:0708.2443 [hep-ph]].

[23] W. Bernreuther, R. Bonciani, T. Gehrmann, R. Heinesch, T. Leineweber, P. Mastrolia and E. Remiddi, Nucl. Phys. B 706, 245 (2005) arXiv:hep-ph/0406046. 


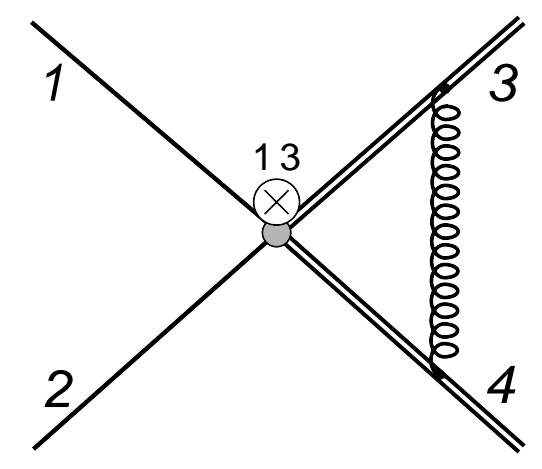




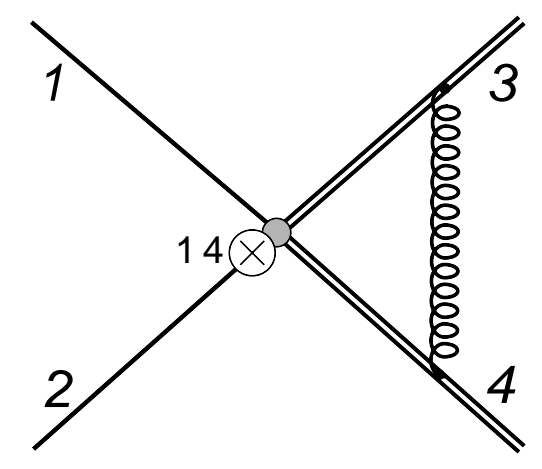

